\title{
Determinants of intrafraction variation during image-guided hypofractionated radiotherapy of the prostate
}

\author{
Chirag Shah • Neelam Tyagi • Inga S. Grills • \\ Larry L. Kestin • Hong Ye - Thomas B. Lanni • Di Yan • \\ Alvaro Martinez $\cdot$ Mihai Ghilezan
}

Received: 15 April 2012 / Accepted: 15 May 2012 /Published online: 9 June 2012

(C) Springer-Verlag 2012

\begin{abstract}
Purpose The purpose of this study is to examine intrafraction variation and residual error (IFV+RE) during conebeam CT (CBCT)-guided hypofractionated prostate radiotherapy and evaluate factors associated along with target margins.

Materials and methods Forty-six patients underwent hypofractionated prostate radiotherapy receiving $64 \mathrm{~Gy}$ in 20 fractions with on-line CBCT correction. Analysis of factors associated with IFV + RE including patient characteristics, treatment characteristics, and organ volumes was performed using univariate and multivariate analyses.

Results The mean IFV + RE were $0.10 \pm 1.1,-1.1 \pm 2.5$, and $-1.5 \pm 2.7 \mathrm{~mm}$ in the mediolateral (ML), anteroposterior (AP), and craniocaudad (CC) dimensions, respectively. The IFV+RE vector was $3.5 \pm 2.5 \mathrm{~mm}$. It was found that 1 , 22 , and $31 \%$ of fractions had an IFV + RE greater than $3 \mathrm{~mm}$ in the ML, $\mathrm{AP}$, and $\mathrm{CC}$ dimensions, respectively. Multivariate analysis found that age and rectal volumes are associated with increased IFV+RE. When evaluating IFV+RE greater than $3 \mathrm{~mm}$, age, treatment time, and rectal volumes were associated. A treatment time of greater than $16.5 \mathrm{~min}$ was found to be a cut point for increased IFV+RE.

Conclusions IFV may represent a significant component of target margins utilized in hypofractionated prostate radiotherapy with target margins exceeding $3 \mathrm{~mm}$ in the $\mathrm{CC}$ and
\end{abstract}

C. Shah $\cdot$ N. Tyagi $(\bowtie) \cdot$ I. S. Grills $\cdot$ L. L. Kestin $\cdot H$. Ye $\cdot$

T. B. Lanni $\cdot$ D. Yan $\cdot$ A. Martinez $\cdot$ M. Ghilezan

Department of Radiation Oncology, William Beaumont Hospital,

3601 West Thirteen Mile Road,

Royal Oak, MI 48073, USA

e-mail: Neelam.Tyagi@beaumont.edu

C. Shah

e-mail: csshah27@hotmail.com
AP dimensions. Rectal volumes, treatment time, and age are associated with IFV+RE.

Keywords Prostate cancer · Hypofractionation · Margins · IGRT

\section{Introduction}

Prostate cancer represents the most common non-cutaneous malignancy in men and the second most common cause of cancer mortality with 186,000 new cases and 29,000 deaths per year [1]. Recently, hypofractionated radiotherapy has emerged as an alternative to conventional dose-escalated treatment. Benefits to this new form of therapy are a reduction in overall treatment duration by more than $50 \%$ and a radiobiological benefit based on an $\alpha / \beta$ of approximately 1.5 for prostate cancer, which could increase biochemical disease-free survival by $15-20 \%$ without changing late toxicity profiles [2]. Initial publications have found comparable to improved acute and late toxicity profiles and promising clinical outcomes with hypofractionated schedules compared to standard fractionation schedules [3].

Developments in treatment delivery and image-guided radiotherapy (IGRT) have allowed for the delivery of a high dose of radiation per fraction utilizing a series of highly conformal beams while still allowing for normal tissue sparing. However, when utilizing these highly conformal treatments, target margins are often reduced and the potential for target miss exists. Further, with the advent of IGRT and the use of on-line conebeam CT (CBCT), target margins can be further reduced. However, these techniques can lengthen overall fraction time, increasing concern for intrafraction variation (IFV) and the potential for target miss due to IFV. IFV has been assessed previously in 
hypofractionated prostate radiation therapy. Analysis of IFV at the University of California San Francisco demonstrated large amplitude sporadic motion, with the authors concluding that on-line target monitoring and correction is necessary [4]. While previous data exists regarding IFV with standard fractionation prostate treatment, the increased treatment times and the use of IGRT limits the ability to extrapolate from these studies. Therefore, the purpose of this analysis was to use data from a prospective trial to assess the magnitude of IFV + residual error (RE), along with predictors of IFV $+\mathrm{RE}$ and margins required during CBCTguided hypofractionated prostate radiation therapy.

\section{Materials and methods}

From November 2007 to July 2010, 46 patients with prostate cancer were enrolled on a prospective phase II in-house institutional review board-approved protocol and underwent hypofractionated prostate radiation therapy delivering 3.2 Gy daily for 20 fractions with daily IGRT over 4 weeks. A total of 920 fractions were delivered and $\mathrm{CBCT}$ measurements were available for 888 of the fractions.

Prior to simulation, patients underwent placement of three 0.35 -mm-diameter/3-cm-length gold Visicoil markers (Core Oncology, Santa Barbara, CA). At simulation, patients underwent a free-breathing $\mathrm{CT}$ in the supine treatment position with immobilization via a custom Alpha Cradle (KGF Enterprises, Chesterfield, MI). Patients were placed on bowel protocol to limit rectal gas prior to simulation but no restrictions on fluid intake were made. In addition to a helical CT scan, all patients underwent a 3T MRI scan. The MRI and CT scans were fused based on registration to the Visicoils. The MRI served as the template for more precise localization of the prostate gland boundaries, seminal vesicles, neurovascular bundles, penile bulb, bladder, and rectum. Risk factors (RF) used in the study included a Gleason score of 7 or higher and a PSA of 10 and higher. The clinical target volume (CTV) expansion was $0 \mathrm{~mm}$ for zero RF, $2 \mathrm{~mm}$ for one RF, and $4 \mathrm{~mm}$ for two RFs [5]. The CTV expansion excluded the bladder and rectum. Planning target volume (PTV) expansions were a 3-mm uniform expansion of the CTV consistent with previous series reporting on hypofractionated prostate radiotherapy [6]. All patients were treated with the Elekta Axesse linear accelerator with onboard KV CBCT (Elekta Oncology Systems, Stockholm, Sweden).

\section{Target localization}

Localization and correction of the target position were performed by registration to the Visicoils from the pre- correction $\mathrm{CBCT}$ to the planning $\mathrm{CT}$ prior to each fraction delivery. Correction was initially performed using the automated soft tissue algorithm with a clip box surrounding the prostate followed by manual adjustments to the Visicoils directly using the X-ray Volume Imaging software (Elekta Oncology Systems, Stockholm, Sweden). No rotational corrections were made; if significant rotation (at discretion of imaging specialist) was identified, patients were re-positioned and CBCT repeated. Of note, no post-correction CBCT was obtained and therefore, when the pre-correction CBCT and post-treatment $\mathrm{CBCT}$ were used for calculation of the IFV, this encompassed the residual error after correction as well as the IFV that occurred during treatment. Following treatment, a post-treatment CBCT was performed. Treatment time was recorded as the time from the beginning of the pre-correction $\mathrm{CBCT}$ to the beginning of the post-treatment CBCT. Retrospectively, in order to determine the association between organ volume and IFV + RE, the prostate, seminal vesicles, rectum, and bladder were retrospectively contoured on both the pre-correction and post-treatment CBCTs for all fractions in 20 patients and volumes of each organ obtained from the simulation, pre-correction CBCT, and post-treatment $\mathrm{CBCT}$. The 20 patients were selected based on availability of all CBCT images on which to contour organ volumes. Due to the image quality limitations of CBCT compared to traditional diagnostic fan beam $\mathrm{CT}$, all contours were placed by a single physicist (NT) and independently verified by a physician (CS), both of whom routinely evaluated CT scans of the abdomen and pelvis.

$\mathrm{IFV}+\mathrm{RE}$ was quantified by assessing the tumor (prostate gland) position in the mediolateral (ML), anteroposterior (AP), and craniocaudad (CC) on the post-treatment CBCT [7]. A composite IFV $+\mathrm{RE}$ value was created using a 3-D vector of the three dimensions as previously utilized [8]. In order to examine the impact of patient/treatment characteristics and organ volumes, univariate and multivariate analyses were performed to evaluate age, weight, treatment time, and prostate/bladder/seminal vesicle/rectal volumes from simulation, pre-correction, and post-treatment. Differences in organ volumes between pre-correction and post-treatment CBCT were analyzed as well.

Target margins were evaluated using the two-parameter model (population mean $\left(M_{\mathrm{p}}\right)$, population standard deviation $\left.\left(\sigma_{\mathrm{p}}\right)\right)$ and the four-parameter model $\left(M_{\mu}\right.$ is the mean of all the patient means, $\Sigma_{\mu}$ is the standard deviation of all of the patient means, root mean square (RMS, $\sigma$ ) is the root mean square of the all the patient standard deviations, $\Sigma_{\sigma}$ is the standard deviation of all the patient standard deviations) based on the post-treatment CBCT [7]. The margin (i.e., CTV to PTV expansion) based on the two-parameter model 
with $90 \%$ confidence was calculated using the following equation:

$M_{x}=M_{\mathrm{p}}+\left(2.5 \times \sigma_{\mathrm{p}}\right)$.

The dosimetric margin using a modified Van Herk's formula based on a four-parameter model with $90 \%$ confidence was calculated using the following equation:

$M_{x}=\left|M_{\mu}\right|+2.5 \sum_{\mu}+1.28\left(\sqrt{ }\left(\left(\operatorname{RMS}(\sigma)+1.3 \sum_{\sigma}\right)^{2}+\sigma_{\mathrm{p}}\right)-\sigma_{\pi p}\right)$

$\sigma_{\mathrm{p}}$ represents the standard deviation of the penumbra in millimeters $[8,9]$. Therefore, these margin parameters account for organ motion based on the shifts in the target position (which organ motion impacts).

Statistical associations between variables were analyzed using Fisher's exact test (two-tailed), logistic regression, and linear regression. Multivariate analysis was also performed following principal component extraction due to correlation between factors in the univariate analysis of IFV+RE. Receiver operating characteristic (ROC) curves were utilized in order to develop cut points for IFV+RE. A $p$ value of $\leq 0.05$ was considered statistically significant. Statistical analyses were performed with SPSS version 17.0.

\section{Results}

The mean IFV+RE vector magnitude of the 888 fractions was $3.51 \pm 2.47 \mathrm{~mm}$ (range, $0-6.5 \mathrm{~mm}$ ). When fractions were analyzed in each dimension the mean and standard deviation was $0.10 \pm 1.1 \mathrm{~mm}$ (range, $0-10.0 \mathrm{~mm}$ ), $-1.1 \pm$ $2.5 \mathrm{~mm}(-11.8-15.1 \mathrm{~mm}$ ), and $-1.5 \pm 2.7 \mathrm{~mm}$ (range, $-10.9-9.9 \mathrm{~mm}$ ) in the ML, AP, and CC, dimensions, respectively. It was found that $1.2,22.2$, and $31.1 \%$ of fractions had an IFV + RE greater than $3 \mathrm{~mm}$ in the $\mathrm{ML}, \mathrm{AP}$, and $\mathrm{CC}$ dimensions, respectively.

Univariate analysis of patient, treatment, and organ volume characteristics was performed relative to the IFV $+\mathrm{RE}$ vector and is presented in Table 1. With regards to patients characteristics, increasing age was significantly associated $(p<0.001)$ while there was a trend for increasing weight $(p=0.08)$. Both increasing treatment time $(p=0.007)$ and afternoon treatment $(p=0.02)$ were significantly associated with IFV + RE. Increasing post-treatment rectal volume compared with simulation scan $(p=0.009)$, increasing intrafraction rectal volume change $(p=0.003)$, simulation bladder volume $(p=0.002)$, and smaller pre-correction and posttreatment bladder volumes were associated with IFV $+\mathrm{RE}$ ( $p=0.01, p=0.02)$. Rectal wall volumes in relation to precorrection and post-treatment rectal volumes were analyzed and were significantly associated $(p=0.002, p<0.001)$. A
Table 1 Univariate analysis of IFV + residual error

\begin{tabular}{|c|c|}
\hline Characteristic & $p$ value \\
\hline Age & $<0.001$ \\
\hline Weight & 0.08 \\
\hline Treatment time & 0.007 \\
\hline Time of day & 0.02 \\
\hline Simulation prostate volume & 0.82 \\
\hline Pre-correction prostate & 0.44 \\
\hline Post-treatment prostate & 0.47 \\
\hline Pre-correction prostate ( $\%$ of simulation) & 0.05 \\
\hline Post-treatment prostate ( $\%$ of simulation) & 0.02 \\
\hline Difference pre-correction vs. post-treatment prostate & 0.49 \\
\hline Simulation seminal vesicle volume & 0.003 \\
\hline Pre-correction seminal vesicle & 0.12 \\
\hline Post-treatment seminal vesicle & 0.47 \\
\hline Pre-correction seminal vesicle ( $\%$ of simulation) & 0.56 \\
\hline Post-treatment seminal vesicle ( $\%$ of simulation) & 0.39 \\
\hline $\begin{array}{l}\text { Difference pre-correction vs. post-treatment seminal vesicle } \\
\text { volume }\end{array}$ & 0.41 \\
\hline Simulation rectal volume & 0.66 \\
\hline Pre-correction rectal & 0.12 \\
\hline Post-treatment rectal & 0.009 \\
\hline Pre-correction rectal (\% of simulation) & 0.36 \\
\hline Post-treatment rectal ( $\%$ of simulation) & 0.03 \\
\hline Difference pre-correction vs. post-treatment rectal volume & 0.003 \\
\hline Rectal wall/pre-correction rectal volume & 0.002 \\
\hline Rectal wall/post-treatment rectal volume & $<0.001$ \\
\hline Simulation bladder volume & 0.002 \\
\hline Pre-correction bladder & 0.01 \\
\hline Post-treatment bladder & 0.02 \\
\hline Pre-correction bladder ( $\%$ of simulation) & 0.13 \\
\hline Post-treatment bladder ( $\%$ of simulation) & 0.19 \\
\hline Difference pre-correction vs. post-treatment bladder volume & 0.54 \\
\hline
\end{tabular}

multivariate analyses (MVA) was performed for factors significant on UVA and is presented in Table 2 with increasing age $(p=0.003)$, increasing post-treatment rectal volume $(p=0.007)$, increasing difference between intrafraction rectal volume change $(p=0.02)$, and decreasing rectal wall/post-treatment rectal volume ratio $(p=0.02)$ being significant. Due to correlation of the variables in the MVA, principle component extraction was performed with four components representing $77 \%$ of the variance: precorrection bladder volume, treatment time, post-treatment prostate volume, and the ratio of rectal wall/posttreatment rectal volume. Multivariate analysis performed utilizing this technique identified pre-correction bladder volume $(p=0.04)$ and rectal wall/post-treatment rectal volume $(p<0.001)$ as significant factors. 
Table 2 Multivariate analysis of IFV + residual error

\begin{tabular}{ll}
\hline Characteristic & $p$ value \\
\hline Age & 0.003 \\
Treatment time & 0.73 \\
Time of day & 0.68 \\
Post-treatment prostate volume change & 0.21 \\
Post-treatment rectal volume & 0.007 \\
Post-treatment rectal volume change & 0.09 \\
Difference pre-correction vs. post-treatment rectal volume & 0.02 \\
Simulation bladder volume & 0.06 \\
Pre-correction bladder volume & 0.51 \\
Post-treatment bladder volume & 0.60 \\
Rectal wall/pre-correction rectal volume & 0.27 \\
Rectal wall/post-treatment rectal volume & 0.02 \\
\hline
\end{tabular}

$\mathrm{IFV}+\mathrm{RE}$ greater than $3 \mathrm{~mm}$

Of the 888 fractions analyzed, 410 fractions (46.2\%) had an $\mathrm{IFV}+\mathrm{RE}$ vector greater than $3 \mathrm{~mm}$ (target margin). Univariate analysis of fractions with an IFV $+\mathrm{RE}$ vector $<3 \mathrm{~mm}$ versus fractions with an IFV $+\mathrm{RE}$ vector $<3 \mathrm{~mm}$ is presented in Table 3 . Increasing age was associated with a vector greater than $3 \mathrm{~mm}$ $(p<0.001)$ as was increasing treatment time $(p<0.001)$ while time of day was not associated $(p=0.29)$. Increasing precorrection rectal volume $(p=0.03)$, post-treatment rectal volume $(p=0.01)$, post-treatment rectal volume change from the simulation scan $(p=0.04)$, and difference in pre-correction and post-treatment rectal volume $(p=0.01)$ were significantly associated with $\mathrm{IFV}+\mathrm{RE}$ vectors greater than $3 \mathrm{~mm}$ with a trend for increasing post-treatment bladder volume $(p=0.06)$. Rectal wall analysis found a decreasing rectal wall/pre-correction rectal volume ratio $(p=0.001)$ and rectal wall/post-treatment rectal volume ratio $(p<0.001)$ to be significantly associated with IFV + RE. Multivariate analysis found only increasing age $(p=0.02)$ to be associated with IFV $+\mathrm{RE}$ greater than $3 \mathrm{~mm}$. Principle component extraction was performed with four components identified: treatment time, pre-correction prostate volume, post-treatment bladder volume, and ratio of rectal wall/post-treatment rectal volume. Multivariate analysis performed utilizing this technique, identified only decreasing rectal wall/post-treatment rectal volume $(p=0.001)$ to be associated with IFV + RE vector greater than $3 \mathrm{~mm}$. ROC curves were created in order to determine cut points predictive of IFV $+\mathrm{RE}$ vector greater than $3 \mathrm{~mm}$ with the results in Table 4. Cut points identified included a treatment time of $16.5 \mathrm{~min}$, pre-correction rectal volume of $51.98 \mathrm{cc}$, pre-correction bladder volume of $94.12 \mathrm{cc}$, and rectal wall/post-treatment rectal volume of 0.27 .

Utilizing post-treatment (IFV $+\mathrm{RE}$ ) CBCT's the margins in the ML, CC, and AP dimensions was 2.6, 7.3, and $8.3 \mathrm{~mm}$ using the two-parameter model and 1.7, 6.6, and $7.3 \mathrm{~mm}$ using the four-parameter model as displayed in Table 5 along with the parameters composing each model.

\section{Discussion}

The results of this study found the mean IFV+RE to be $0.10 \pm 1.05,-1.14 \pm 2.50$, and $-1.52 \pm 2.73 \mathrm{~mm}$ in the ML,
Table 3 Analysis of IFV+residual error greater than or less than $3 \mathrm{~mm}$ (mean values)

\begin{tabular}{lclc}
\hline Characteristic & $p$ value & Characteristic & $p$ value \\
\hline Age & $<0.001$ & Rectal volumes & \\
Weight & 0.45 & Simulation & 0.87 \\
Treatment time & 0.001 & Pretreatment & 0.03 \\
Prostate volumes & & Post-treatment & 0.006 \\
Simulation & 0.88 & Pre difference (vs. simulation) & 0.26 \\
Pre-correction & 0.63 & Post difference (vs. simulation) & 0.04 \\
Post-treatment & 0.68 & Pre-correction vs. post-treatment & 0.01 \\
Pre-correction difference (vs. simulation) & 0.04 & Wall/pre volume & 0.001 \\
Post difference (vs. simulation) & 0.03 & Wall/post volume & $<0.001$ \\
Pre-correction vs. post-treatment difference & 0.51 & Bladder volumes & \\
Seminal vesicle volumes & & Simulation & 0.17 \\
Simulation & 0.14 & Pre-correction & 0.08 \\
Pre-correction & 0.08 & Post-treatment & 0.06 \\
Post-treatment & 0.57 & Pre-correction difference (vs. & 0.58 \\
Pre-correction difference (vs. simulation) & 0.75 & Post-treatment difference (vs. simulation) & 0.81 \\
Post-treatment difference (vs. simulation) & 0.47 & Pre-correction vs. post-treatment & 0.99 \\
Pre-correction vs. post-treatment difference & 0.46 & difference & \\
\hline
\end{tabular}


Table 4 Cut points for IFV+residual error greater than $3 \mathrm{~mm}$

\begin{tabular}{lccc}
\hline Parameter & Cut point & Sensitivity, \% & Specificity, \% \\
\hline Treatment time & $16.5 \mathrm{~min}$ & 67.1 & 42.7 \\
Pre-correction rectal volume & $51.98 \mathrm{cc}$ & 71.6 & 38.9 \\
Pre-correction bladder volume & $94.12 \mathrm{cc}$ & 58.6 & 58.2 \\
Post-treatment rectal volume & $57.08 \mathrm{cc}$ & 64.7 & 46.7 \\
Post-treatment bladder volume & $112.29 \mathrm{cc}$ & 56.7 & 59.3 \\
Rectal wall/post-treatment rectal & 0.27 & 71.2 & 24.6 \\
$\quad$ & & & \\
\hline
\end{tabular}

$\mathrm{AP}$, and $\mathrm{CC}$ dimensions, respectively, with a mean 3-D vector of $3.51 \pm 2.47 \mathrm{~mm}$. More importantly, $46 \%$ of fractions had a vector greater than $3 \mathrm{~mm}$, the standard PTV expansion utilized in our study. The IFV+RE measured in this study are consistent with data previously published and represent the largest cohort of hypofractionated patients on which IFV has been measured. Unfortunately, the majority of previous IFV data has been recorded on patients receiving standard fractionation radiation therapy; the longer treatment times associated with hypofractionated treatment may limit the ability to extrapolate from these studies as data from Stanford University demonstrated that with increasing time from correction, there are an increasing percentage of fractions that exceed a 2-mm margin [10]. Also, previous studies often utilized 2-D orthogonal imaging-based measurements rather than true 3-D imaging. However, a recent series from Belgium evaluated 27 patients receiving standard fractionation with IGRT and found IFV to be $0.1 \pm 1.1,2.3 \pm 1.5$, $0.2 \pm 1.1 \mathrm{~mm}$ in the ML, AP, and CC dimensions, respectively, with the ML component being similar, the AP component being larger, and $\mathrm{CC}$ component smaller than our analysis [11]. The largest series of standard fractionated patients

Table 5 Target margins utilizing the two- and four-parameter models based on pre- and post-treatment cone beam CT scans

\begin{tabular}{lccc}
\hline & \multicolumn{2}{l}{ post-treatment } & \\
\cline { 2 - 4 } Two-parameter & ML & CC & AP \\
\hline$M_{\mathrm{p}}$ & 0.01 & -0.11 & -0.15 \\
$\sigma_{\mathrm{p}}$ & 0.10 & 0.25 & 0.27 \\
Margins (mm) & 2.6 & 7.3 & 8.3 \\
Four-parameter & $\mathrm{ML}$ & $\mathrm{CC}$ & $\mathrm{AP}$ \\
$M_{\mu}$ & 0.01 & -0.11 & -0.15 \\
$\Sigma_{\mu}$ & 0.06 & 0.15 & 0.18 \\
$\Sigma_{\sigma}$ & 0.02 & 0.08 & 0.08 \\
RMS $(\sigma)$ & 0.08 & 0.20 & 0.21 \\
Margins $(\mathrm{mm})$ & 1.7 & 6.6 & 7.3 \\
\hline
\end{tabular}

Abbreviations: $M_{p}$ populations mean, $\sigma_{p}$ populations standard deviation, $M_{\mu}$ mean of all patient means, $\Sigma_{\mu}$ standard deviation of the patient means, $\Sigma_{\sigma}$ standard deviation of the standard deviation for each patient, $R M S(\sigma)$ root mean square of $\sigma$ evaluated included 427 patients (11,426 fractions) receiving prostate intensity-modulated radiotherapy (IMRT) and found IFV was larger than $2 \mathrm{~mm}$ in $66 \%$ of fractions [12].

The current study identified patient, treatment, and organ volume characteristics that were associated with IFV. Our analysis of IFV+RE greater than $3 \mathrm{~mm}$ found that age and treatment time, as well as organ volumes were associated. Age may be associated to older patients' inability to stay in position as well as that of younger patients or may reflect factors not accounted for on the univariate and multivariate analyses. Rectal volumes were found to be a significant factor associated with IFV $+\mathrm{RE}$ and IFV $+\mathrm{RE}$ greater than $3 \mathrm{~mm}$ despite employing a rectal protocol for patients. Additional interventions may need to be taken (ex. Endorectal balloon) to eliminate this risk factor in future. Another finding from our study is that cut points predictive of IFV+ $\mathrm{RE}$ larger than the 3-mm expansion were delineated. To our knowledge, this data represents the only data to analyze the impact of these factors on IFV. While treatment time has been found to be associated with IFV in prostate cancer treatment as well as in other sites, limited data has been presented on organ volume and treatment time cut points to help create an adaptive management protocol for IFV. Going forward, clinicians may utilize patient simulation or precorrection on-line organ volumes and treatment times to identify patients at risk for large IFV and make appropriate changes to PTV margins in an adaptive fashion based on the initial fractions delivered. The benefit of this strategy over simply creating a population-based margin created from a large number of IFV values is that it allows for reduction of margins in patients who are likely to have low IFV and therefore, decreased risk of normal tissue complications. However, at this time this data needs to be validated and criteria further refined as the specificity of our targets remains low.

Multiple series have examined margin determination based on IFV and the results are comparable to our findings in the ML dimension, but our study found much larger margins in the CC, and AP dimensions. A study of 17 patients undergoing standard fractionated prostate radiation imaged patients at 8-min intervals following on-line corrections. The results of this study were that with on-line correction, target margins required to compensate for IFV were $1.1,1.8$, and $2.3 \mathrm{~mm}$ in the ML, SI, and AP dimensions, respectively [13]. A larger series of 40 patients $(1,532$ fractions) were evaluated by four localization methods: skin based, bony anatomy, implanted seeds with $5 \mathrm{~mm}$ threshold, and implanted seeds with no threshold. Utilizing the no threshold localization, target margins were $3.4,3.2$, and $3.2 \mathrm{~mm}$ in the ML, CC, and AP dimensions, respectively [14]. A series of 14 patients receiving prostate IMRT with on-line CBCT correction based on three implanted transponders found that margins required were 1.4, 2.6, and $2.3 \mathrm{~mm}$ in the $\mathrm{ML}, \mathrm{CC}$, and $\mathrm{AP}$ dimensions, respectively, 
with traditional dose per fractions utilized [15]. Further, as mentioned previously, analysis of 47 patients receiving hypofractionated prostate radiation therapy with implanted fiducials found target margins of $1.4,1.9$, and $2.0 \mathrm{~mm}$ in the $\mathrm{ML}, \mathrm{AP}$, and $\mathrm{CC}$ dimensions, respectively, using portal imaging [16]. This increase in margins from our data may be secondary to the addition of residual error into our IFV calculations; however, residual error must be incorporated into margin calculations as a non-zero residual must be accounted for in a PTV expansion. Also, another significant finding in our study was the reduction in target margins with on-line CBCT IGRT compared to no IGRT. Target margins without IGRT were $9.6-12.2 \mathrm{~mm}$ without IGRT compared with $2.6-8.3 \mathrm{~mm}$ with IGRT.

Multiple solutions have been presented to accommodate for IFV while maintaining reasonable target margins for hypofractionated schedules. Analysis of correction of IFV at each field delivery has found that this strategy reduces the time with IFV greater than $3 \mathrm{~mm}$ by more than $50 \%$ (10 vs. $23 \%$ ) [17]. This is further justified by data from the University of Michigan which found that target margins were reduced by on-line correction between beams and further reduced by on-line correction during each individual beam [18]. Also, techniques such as real-time tumor position tracking with using an electromagnetic system may provide a means to minimize IFV and its impact on target margins [19]. Another alternative is to pursue an adaptive management protocol that bases margins on individual specific assessment of IFV. The feasibility of such methods has been documented but limited data is available [20]. Finally, changing delivery techniques using arc therapy or high output accelerators can reduce treatment time and therefore IFV.

There are limitations of this study: the extent of IFV+RE and margins may be overestimated based on post-treatment CBCT due to the time lag from completion of treatment to imaging. With newer techniques such as electromagnetic tracking, more accurate calculations may be made. In addition, the impact of rotational correction on target margins and treatment parameters was not evaluated in this study and is currently under investigation. Also, the geometric and dosimetric margin formula assumes infinite fractions which may need to be further revised when utilizing hypofractionated schedules. Finally, our calculations were based on IFV $+\mathrm{RE}$ which may make comparison to previous studies more difficult.

\section{Conclusions}

The results presented herein illustrate that IFV + RE may represent a significant component of the target margin in a subset of patients receiving hypofractionated prostate radiotherapy. Rectal volumes are the factor most associated with IFV $+\mathrm{RE}$ exceeding the target margin of $3 \mathrm{~mm}$.

Conflict of interest No conflict of interest.

\section{References}

1. Jemal A, Siegel R, Ward E et al (2006) Cancer statistics, 2006. CA Cancer J Clin 56:106-130

2. Fowler JF, Ritter MA, Chappell RJ et al (2003) What hypofractionated protocols should be tested for prostate cancer? Int J Radiat Oncol Biol Phys 56:1093-1104

3. Kupelian PA, Willoughby TR, Reddy CA, Klein EA, Mahadevan A (2007) Hypofractionated intensity modulated radiotherapy (70 Gy at 2.5 Gy per fraction) for localized prostate cancer: Cleveland Clinic experience. Int J Radiat Oncol Biol Phys 68:1424-1430

4. Hossain S, Xia P, Chuang C et al (2008) Simulated real time image guided intrafraction tracking-delivery for hypofractionated prostate IMRT. Med Phys 35:4041-4048

5. Kestin L, Goldstein N, Vicini F et al (2002) Treatment of prostate cancer with radiotherapy: should the entire seminal vesicles be included in the clinical target volume? Int J Radiat Oncol Biol Phys 54:686-697

6. Boike TP, Lotan Y, Cho LC et al (2011) Phase I dose-escalation study of stereotactic body radiation therapy for low- and intermediate-risk prostate cancer. J Clin Oncol 29:2020-2026

7. Yan D, Lockman D, Martinez A (2005) Computed tomography guided management of interfractional patient variation. Semin Radiat Oncol 15:168-179

8. Purdie TG, Bissonnette JP, Franks K et al (2007) Cone-beam computed tomography for on-line image guidance of lung stereotactic radiotherapy: localization, verification, and intrafraction tumor position. Int J Radiat Oncol Biol Phys 68:243-252

9. van Herk M, Remeijer P, Rasch C, Lebesque JV (2000) The probability of correct target dosage: dose-population histograms for deriving treatment margins in radiotherapy. Int J Radiat Oncol Biol Phys 47:1121-1135

10. Xie Y, Djajputra D, King CR et al (2008) Intrafractional motion of the prostate during hypofractionated therapy. Int J Radiat Oncol Biol Phys 72:236-246

11. Budhiharto T, Slagmolen P, Haustermans K et al (2011) Intrafractional prostate motion during online image guided intensitymodulated radiotherapy for prostate cancer. Radiother Oncol 98:181-186

12. Kotte AN, Hofman P, Lagendijk JJ et al (2007) Intrafraction motion of the prostate during external-beam radiation therapy: analysis of 427 patients with implanted fiducial markers. Int $\mathbf{J}$ Radiat Oncol Biol Phys 69:419-425

13. Su Z, Zhang L, Murphy M, Williamson J (2011) Analysis of prostate patient setup and tracking data: potential intervention strategies. Int J Radiat Oncol Biol Phys 81:880-887

14. Beltran C, Herman MG, Davis BJ (2008) Planning target margin calculations for prostate radiotherapy based on intrafraction and interfraction motion using four localization methods. Int J Radiat Oncol Biol Phys 70:289-295

15. Tanyi JA, He, Tongming H, Summers PA et al (2010) Assessment of planning target volume margins for intensity- 
modulated radiotherapy of the prostate gland: role of daily inter- and intrafraction motion. Int J Radiat Oncol Biol Phys 78:1579-1585

16. Madsen BL, Hsi RA, Pham HT et al (2003) Intrafractional stability of the prostate using a stereotactic radiotherapy technique. Int $\mathrm{J}$ Radiat Oncol Biol Phys 57:1285-1291

17. Liu W, Qian J, Hancock SL, Xing L, Luxton G (2010) Clinical development of failure detection-based online repositioning strategy for prostate IMRT - experiments, simulation, and dosimetry study. Med Phys 37:5287-5297
18. Litzenberg DW, Balter JM, Hadley SW et al (2006) Influence of intrafraction motion on margins for prostate radiotherapy. Int $\mathrm{J}$ Radiat Oncol Biol Phys 65:548-553

19. Abdel-Wahab M, Mahmoud O, Merrick G et al (2012) ACR appropriateness criteria external-beam radiation therapy treatment planning for clinically localized prostate cancer. J Am Coll Radiol 9:233-238

20. Adamson J, Wu Q, Yan D (2011) Dosimetric effect of intrafraction motion and residual setup error for hypofractionated prostate intensitymodulated radiotherapy with online cone beam computed tomography image guidance. Int J Radiat Oncol Biol Phys 80:453-461 